\title{
Barbiturates as Noncompetitive Inhibitors of PTP1B and Competitive Inhibitors of Vaccinia H1-Related (VHR) Phosphatase ${ }^{\dagger}$
}

\author{
Deegendra Khadka, Bhooshan Kafle, Hwangseo Park, ${ }^{\star}$ and Hyeongjin Cho \\ Department of Chemistry, College of Natural Sciences, Inha University, Incheon 402-751, Korea \\ *E-mail: hcho@inha.ac.kr \\ ${ }^{\star}$ Department of Bioscience and Biotechnology, College of Engineering, Sejong University, Seoul 143-747, Korea \\ Received March 4, 2011, Accepted April 5, 2011
}

Key Words : Barbiturate, Protein tyrosine phosphatase, Vaccinia H1-related, VHR, PTP1B

Barbiturates have a long history of being used as drugs. Since the first barbiturate, Veronal, was marketed as a sleeping aid more than a century ago, barbiturates have been used as central nervous system (CNS) depressants. ${ }^{1}$ Short-acting barbiturates are used as anesthetics and long-acting ones as antiepilepsy drugs. ${ }^{2}$ Sodium pentothal belongs to the first class and phenobarbital to the latter class. ${ }^{2}$ Beside the effects on the CNS, wide varieties of other biological activities have been recognized for barbiturates. ${ }^{3}$ Noteworthy among them is the inhibition of the phosphatase activity of calcineurin, a protein Ser/Thr phosphatase. ${ }^{4}$

Recently, we reported the inhibitory activities of barbiturates against protein tyrosine phosphatase (PTP) 1B and other PTPs. ${ }^{3}$ The study focused on the barbiturates with a substituent at C-5 of a barbituric acid core structure, and compound 1 was identified as the most potent PTP1B inhibitor among the series. Commonly used barbiturates like phenobarbital and sodium pentothal have a substituent at C-5 of this basic skeleton. In a virtual screening to find a lead compound for PTP1B inhibitor development, we previously identified barbiturates, 2 and $\mathbf{3}$, which showed $\mathrm{IC}_{50}$ values against PTP1B of $35 \mu \mathrm{M}$ and $12 \mu \mathrm{M}$, respectively. ${ }^{5}$ Based on this result, we extended our study to the barbiturates possessing dual substituents at N-1 and C-5 of the barbituric acid core structure.

Barbituric acid derivatives were synthesized by the condensation of $N$-substituted barbituric acid derivatives (A) and $\mathrm{N}$ substituted indole-3-carboxaldehyde derivatives (B) (Scheme 1). ${ }^{6}$ An aldehyde derivative, $\mathbf{B}$ (0.05 mmole), was added as a solid into a solution of $N$-substituted barbiturate $\mathbf{A}(0.05$ mmole $)$

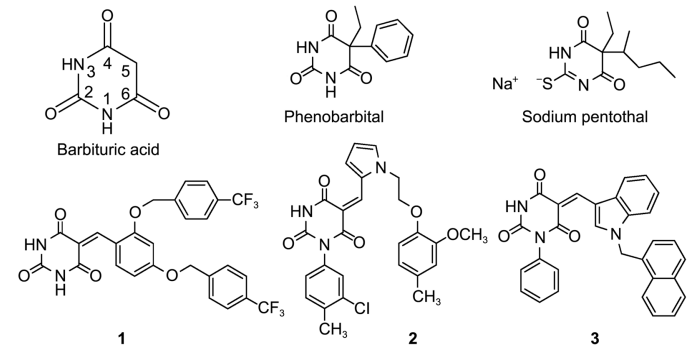

Figure 1. Barbiturates currently used as CNS depressants or mentioned in this study.

This paper is dedicated to Professor Eun Lee on the occasion of his honourable retirement. in dry DMF $(0.1 \mathrm{~mL})$ in an $1.6 \mathrm{~mL}$ sized Eppendorf tube. When heated at $80^{\circ} \mathrm{C}$ for $6 \mathrm{~h}$ and cooled to room temperature, the reaction product precipitated. The precipitate was washed with $i$ PrOH $(0.1 \mathrm{~mL} \times 3)$ to obtain a solid product free from DMF. The products obtained in this way showed essentially a single spot in TLC analysis and were used as such for enzyme experiments without further purification steps. Using this method, multiple condensation reactions could be performed at the same time and the products could be harvested on the same day.

The precursors, $\mathbf{A}$ and $\mathbf{B}$, for the condensation reaction were prepared as shown in Scheme 2. Briefly, $N$-substituted barbiturates (A) were prepared by the conversion of appropriate arylamines (D) to arylurea $(\mathbf{E}),{ }^{7}$ followed by condensation with malonate. ${ }^{6}$ To prepare $N$-substituted indole-3carboxaldehydes (B), indole was treated with appropriate benzylhalide or halomethylnaphthyl derivatives $(\mathbf{F})^{8}$ in the presence of $\mathrm{KOH}$ as a base. ${ }^{9}$

The first set of compounds, 3-9, was prepared with $\mathrm{R}_{1}$ fixed as $\mathrm{H}$ and $\mathrm{R}_{2}$ being varied as shown in Table 1 . These compounds were examined for their inhibitory activity against PTP1B and VHR phosphatase. $\mathrm{IC}_{50}$ values were determined by measuring the enzyme activity at a range of different inhibitor concentrations using $p$-nitrophenyl phosphate ( $p$ NPP) as the substrate. Among the various substituted phenyl and naphthyl derivatives, compound $\mathbf{3}$ and $\mathbf{7}$ with a 1-naphthyl and a 1-(4-bromo)naphthyl moiety, respectively, for $\mathrm{R}_{2}$ gave the lowest $\mathrm{IC}_{50}$ value against PTP1B. From the two, the 1-naphthyl moiety was selected for $\mathrm{R}_{2}$ due to its molecular weight being lower than that of the corresponding bromonaphthyl moiety. A second set of compounds 10-17 was then prepared with $R_{2}$ fixed as the 1-naphthyl moiety. Various derivatives of A were subjected to condensation reactions with 1-naphthyl-B as the counterpart. Among the compounds synthesized, 7 proved to be the most potent inhibitor of PTP1B.

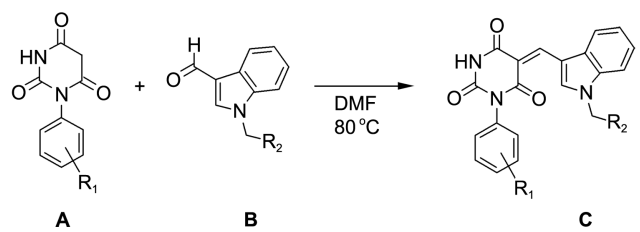

Scheme 1. Synthetic strategy for the barbituric acid derivatives. 
The nature of inhibition by compound 7 was investigated by steady-state kinetic experiments on two human PTPs, PTP1B and VHR phosphatase, and a yeast PTP, YPTP1. PTP1B and YPTP1 are tyrosine-specific PTPs, while VHR phosphatase is classified as a tyrosine and serine/threonine dual-specificity phosphatase, which has little homology with the tyrosine-specific PTPs except at the active site region. ${ }^{10}$ VHR phosphatase is also much smaller in size compared to the catalytic domain of PTP1B. Lineweaver-Burk plot analysis revealed that compound 7 inhibited PTP1B in a noncompetitive fashion (Figure 2a). Noncompetitive inhibition was also observed for YPTP1 (data not shown). On the other hand, compound 7 exhibited a competitive inhibition pattern for VHR phosphatase, which indicates that the inhibitor competes with the substrate in binding on VHR phosphatase (Figure 2b). These results from kinetic experiments suggest the binding of 7 in the active site on VHR phosphatase, but not in the active sites on PTP1B and YPTP1. However, this supposition apparently contradicts the structural diversity of the three PTPs except for at their active sites, especially

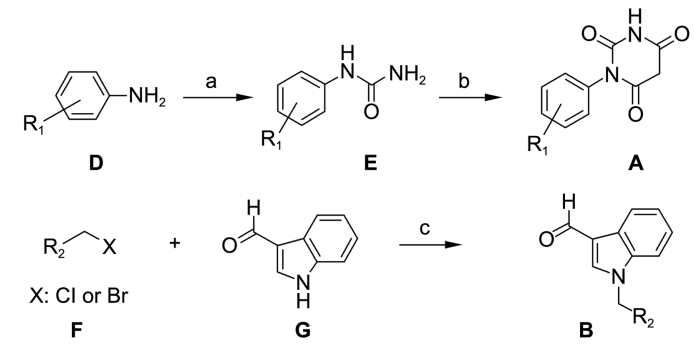

Scheme 2. Synthetic strategy for $N$-substituted barbiturates (A) and $\mathrm{N}$-substituted indole-3-carboxaldehydes (B). Reagent and conditions: (a) $\mathrm{NaOCN}, \mathrm{AcOH} / \mathrm{H}_{2} \mathrm{O}, 37^{\circ} \mathrm{C}$; (b) diethyl malonate, $\mathrm{Na} /$ $\mathrm{EtOH}, 70^{\circ} \mathrm{C}$; (c) $\mathrm{KOH}$, DMSO, rt.

Table 1. Inhibitory activity of the barbituric acid derivatives against PTP1B and VHR phosphatase

\begin{tabular}{ccccc}
\hline Compds. & $\mathrm{R}_{1}$ & $\mathrm{R}_{2}$ & $\begin{array}{c}\mathrm{IC}_{50} \\
\mathrm{PTP1B} \\
(\mu \mathrm{M})^{a}\end{array}$ & $\begin{array}{l}\mathrm{IC}_{50} \\
(\mu \mathrm{M})^{a}\end{array}$ \\
\hline $\mathbf{3}$ & $\mathrm{H}$ & 1-naphthyl & $12 \pm 2$ & $43 \pm 1$ \\
$\mathbf{4}$ & $\mathrm{H}$ & phenyl & $28 \pm 4$ & $66 \pm 6$ \\
$\mathbf{5}$ & $\mathrm{H}$ & 3-chlorophenyl & $23 \pm 4$ & $45 \pm 10$ \\
$\mathbf{6}$ & $\mathrm{H}$ & 1-(4-methoxy)naphthyl & $14 \pm 1$ & $19 \pm 0$ \\
$\mathbf{7}$ & $\mathrm{H}$ & 1-(4-bromo)naphthyl & $11 \pm 0$ & $27 \pm 2$ \\
$\mathbf{8}$ & $\mathrm{H}$ & 2-naphthyl & $24 \pm 2$ & $46 \pm 9$ \\
$\mathbf{9}$ & $\mathrm{H}$ & 4-methoxyphenyl & $25 \pm 3$ & $66 \pm 11$ \\
$\mathbf{1 0}$ & 4-methoxy & 1-naphthyl & $28 \pm 1$ & $45 \pm 1$ \\
$\mathbf{1 1}$ & 3-NO & 1-naphthyl & $14 \pm 3$ & $24 \pm 1$ \\
$\mathbf{1 2}$ & 3-Cl & 1-naphthyl & $18 \pm 1$ & $36 \pm 2$ \\
$\mathbf{1 3}$ & 3-CF & 1-naphthyl & $18 \pm 3$ & $25 \pm 3$ \\
$\mathbf{1 4}$ & 4-F & 1-naphthyl & $24 \pm 2$ & $25 \pm 7$ \\
$\mathbf{1 5}$ & 3- methoxy & 1-naphthyl & $25 \pm 2$ & $33 \pm 1$ \\
$\mathbf{1 6}$ & 4-(4-chlorophenoxy) & 1-naphthyl & $14 \pm 2$ & $31 \pm 2$ \\
$\mathbf{1 7}$ & 3,5-(CF $\mathrm{CF}_{3}$ & 1-naphthyl & $16 \pm 8$ & $19 \pm 1$ \\
\hline
\end{tabular}

${ }^{a}$ Values are the mean \pm standard deviations of two or more experiments. Phosphatase assays were performed, as previously described, using $p \mathrm{NPP}$ as the substrate. ${ }^{3}$ Enzyme concentrations in the assay mixture were $40 \mathrm{nM}$ for PTP1B and $500 \mathrm{nM}$ for VHR phosphatase. $\mathrm{IC}_{50}$ values were determined by measuring the enzyme activity across a range of different inhibitor concentrations. Kinetic data were analyzed using the GraFit 5.0 program (Erithacus Software).
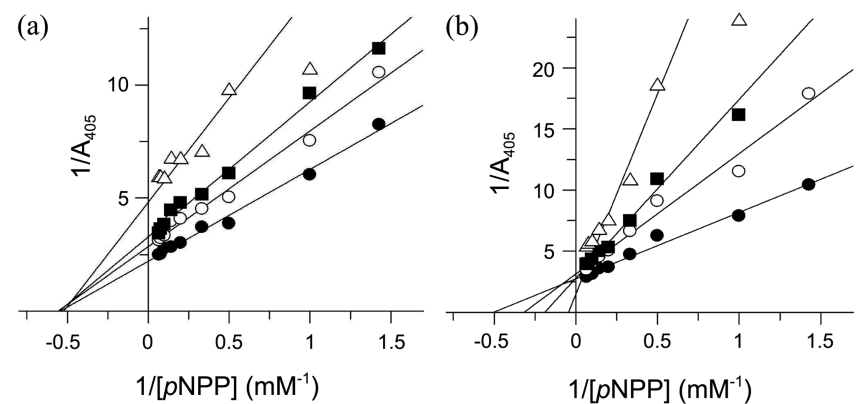

Figure 2. Lineweaver-Burk analysis for PTP1B and VHR phosphatase catalyzed reaction in the presence of compound 7. Phosphatase activity was measured against $p$ NPP for (a) PTP1B in the presence of none $(\bigcirc), 25 \mu \mathrm{M}(\bullet), 50 \mu \mathrm{M}(\square)$, and $100 \mu \mathrm{M}(\triangle)$ of 7 and for (b) VHR phosphatase in the presence of none ( $\bigcirc), 25 \mu \mathrm{M}(\bullet)$, $50 \mu \mathrm{M}(\square)$, and $100 \mu \mathrm{M}(\triangle)$ of 7 .

between VHR phosphatase and the other PTPs. Furthermore, the nondiscriminatory inhibition of the PTPs by compound 7 implies that there exists structural similarity between the inhibitor binding sites on the three PTPs. To the best of our knowledge on the 3-dimensional structures of the PTPs, the active sites of the three PTPs are the only conceivable binding pockets for compound 7 , except the secondary aryl phosphate-binding site present near the active site of PTP1B. In our previous study on monosubstituted barbiturate compounds, similarly conflicting results were obtained. Compound $\mathbf{1}$ exhibited a noncompetitive inhibition pattern for PTP1B and a mixed-type noncompetitive pattern for YPTP $1 .{ }^{3}$ The binding sites of the barbiturate derivatives on PTP1B and YPTP1 are yet to be determined through X-ray crystallographic study of the enzyme-inhibitor complex. However, in the present study, the barbiturate moiety was recognized as a scaffold for the design of active site-direct inhibitors of VHR phosphatase.

Acknowledgments. This research was supported by Inha University.

\section{References}

1. Ho, I. K.; Harris, R. A. Ann. Rev. Pharmacol. Toxicol. 1981, 21, 83.

2. Rosenfeld, G. C., Loose, D. S., Eds.; Pharmacology, 3rd ed.; Lippincott Williams \& Wilkins: Baltimore, 2007; pp 101-147.

3. Kafle, B.; Bhattarai, B. R.; Cho, H. Bull. Korean Chem. Soc. 2011, 32, 31 and references cited therein.

4. Humar, M.; Pischke, S. E.; Loop, T.; Hoetzel, A.; Schmidt, R.; Klaas, C.; Pahl, H. L.; Geiger, K. K.; Pannen, B. H. J. Mol. Pharmacol. 2004, 65, 350.

5. Park, H.; Bhattarai, B. B.; Ham, S. W.; Cho, H. Eur. J. Med. Chem. 2009, 44, 3280.

6. Brückmann, G.; Isaacs, S. D. J. Am. Chem. Soc. 1949, 71, 390.

7. Laudien, R.; Mitzner, R. J. Chem. Soc., Perkin Trans. 2001, 2, 2226.

8. 1-(Bromomethyl)-4-methoxynapthalene was prepared by the reduction of 4-methoxy-1-napthaldehyde by $\mathrm{NaBH}_{4}$, followed by the reaction with $\mathrm{PBr}_{3}$ (Can. J. Chem. 1981, 59, 2629). 1-Bromo4-(bromomethy)naphthalene was synthesized by the bromination of 1-bromo-4-methylnapthalene with $N$-bromosuccinimide in the presence of 2,2-azobis(2-methylpropionitrile) as a catalyst (Tetrahedron Lett. 1995, 36, 3103). All other arylamines (D) and halomethyl derivatives (F) were purchased from Aldrich or TCI.

9. Santoso, M. J. Mat. \& Sains 2000, 5, 91.

10. Yuvaniyama, J.; Denu, J. M.; Dixon, J. E.; Saper, M. A. Science 1996, 272, 1328. 